\title{
Development of methods for performing the maximum voluntary contraction (MVC) test
}

\author{
Mateusz Kukla ${ }^{1 *}$, Bartosz Wieczorek ${ }^{1}$, tukasz Warguła ${ }^{1}$ \\ ${ }^{1}$ Poznań University of Technology, Chair of Basics of Machine Design, 3 Piotrowo St., \\ 60-965 Poznań, Poland
}

\begin{abstract}
The aim of the study is to determine the most effective isometric exercises required in order to perform MVC (Maximum Voluntary Contraction) tests under given static muscle contraction conditions. To this end, a group of four muscles was defined and on this basis, a series of exercises was selected. During electromyography studies, the electrical activity of the muscles was recorded. The article presents the results obtained for all exercises in the whole group of subjects. The data obtained provide a basis for evaluating and selecting the method of loading the analysed muscles during MVC tests. This is necessary in order to perform a normalization of raw (unprocessed) EMG signal, thus enabling comparison of results between individual subjects. Analyses concerning this kind of diagnosis of the electrical activity of muscles are widely applied in research related to rehabilitation and medicine, sports science, but also ergonomics.
\end{abstract}

Keywords: surface electromyography (EMG), maximum voluntary contraction (MVC) test, normalization

\section{Introduction}

Kinesiological electromiography, also called surface electromiography, is an experimental technique for examining the electrical activity of muscles. It involves detecting differences in the potentials of electromagnetic fields generated by the body during muscle contraction. The electrical voltage value determined in this way depends, among other things, on the load that is being overcome. This method of analysis allows to define the activity of selected muscle groups over time during performance of specific exercises. The character of the measurement, however, does not allow to determine the force generated by a given muscle or a number of muscle fibres that become activated. Also, it is impossible to make a straightforward comparison between the values measured in individual measurement sites or for individual persons. This is due to constantly changing physico-chemical conditions in the human body. Despite these limitations surface electromiography is frequently used in research related to rehabilitation and medicine. The methods described are also applied in the engineering practice. Due to their non-invasive character they are in common use in studies within the field of ergonomics, biomechanics or design of rehabilitation equipment

\footnotetext{
${ }^{*}$ Corresponding author: E-mail address: mateusz.kukla@put.poznan.pl, Tel.: +48 612244514

Reviewers: Gregorz Domek, Marcin Kubiak
} 
such as wheelchairs $[1,2]$. They are also widely used for determining muscle strength during performance of various physical activities [3, 4].

The works under this project pertain to the biomechanics of manual wheelchair propulsion. Their outcome will be a basis for the design of new types of manual and hybrid wheelchair drive systems. To achieve this objective a number of secondary tasks were defined, including tasks related to the analysis of muscular activity during wheelchair propulsion. Depending on what users they are intended for, individual types of wheelchair drive systems differ with the source of driving energy and with design. The surface electromiography methods enable comparative analysis of the physical effort required to propel different types of wheelchairs.

\section{Methods}

The study was conducted based on the SENIAM project [5] methodology and information provided in the "The ABC of EMG" manual [6]. Signals were recorded using Noraxon Myotrace 400 apparatus and analysed with MyoResearch XP MT400 4CH Clinical Edition software. The research was conducted on a group of nine subjects characterized in Table 1.

The research methodology involved an analysis of the upper extremity muscles participating in propelling the wheelchair by means of pushrims. Measurements were made on the following muscles: deltoid muscle - anterior and posterior fibres, triceps brachii and extensor carpi radialis longus. After determining the electrode placement sites (Figure 1), the skin surface was prepared by degreasing and hair removal. The purpose of the preparation was to improve the transfer and electrical measurements conditions, thus improving the impedance conditions. In the next step $20 \mathrm{~mm}$ diameter round electrodes were attached in the area of the centres of the bellies of selected muscles at the distance of approx. $20 \mathrm{~mm}$ from each other. An example of the layout of electrodes on one of the subjects is represented in Figure 1. Next the impedance test was performed and if the result was positive (a value below $15 \mathrm{k} \Omega$ was obtained) the research proper was started.

Table 1. Study subjects' characteristics

\begin{tabular}{|c|c|c|c|}
\hline No. & Sex & BMI $\left[\mathbf{k g} / \mathbf{m}^{\mathbf{2}}\right]$ & Height $[\mathbf{c m}]$ \\
\hline I & $\mathrm{F}$ & 19.8 & 162 \\
\hline II & $\mathrm{M}$ & 26.6 & 169 \\
\hline III & $\mathrm{M}$ & 22.3 & 182 \\
\hline IV & $\mathrm{F}$ & 20.4 & 173 \\
\hline V & $\mathrm{M}$ & 26.4 & 173 \\
\hline VI & $\mathrm{F}$ & 20.7 & 166 \\
\hline VII & $\mathrm{M}$ & 21.7 & 173 \\
\hline VIII & $\mathrm{F}$ & 22.4 & 169 \\
\hline IX & $\mathrm{M}$ & 35.0 & 169 \\
\hline
\end{tabular}

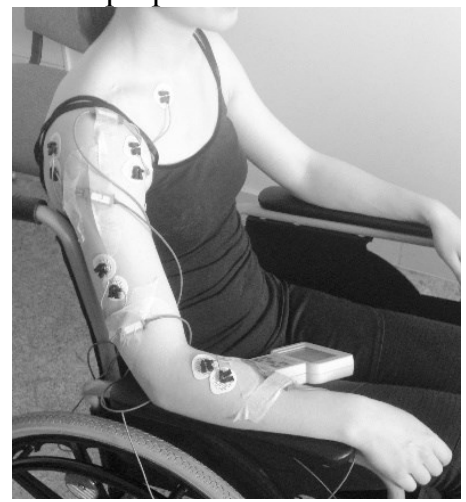

Fig. 1. An example of electrode layout

The study's target group is the disabled. For this reason, a number of important conditions must be taken into consideration when planning future studies of a similar type. To normalize the results popular weight training exercises were chosen and modified in such a way that they could be performed by a subject seated in a wheelchair. The advantage of this choice is the simplicity of the technique of individual exercises and availability of a wide body of practical materials referring to them. Where possible, exercises involving both arms at the same time were chosen. The aim was to attempt to minimize the risk of compensation of the loaded extremity with muscles in other parts of the body, for instance, through shoulder balancing or incorrect position of the back. The measurement taking cycle 
included the following subsequent stages: zeroing of the measurement at the starting position, start of measurement, start of movement - light load on the patient's muscles during performance of the exercise, end of movement - full load on the patient's muscles at the exercise's terminal position, retaining the state of full load over 3 to 5 seconds, release of the load. Next the cycle of the exercise was repeated 3 to 5 times including the intervals, and the measurement was ended. The tests were conducted under the static contraction conditions, with manual loading of individual groups of muscles. Intermediate elements, for instance an element simulating a barbell, with possibly the lowest weight were used at the process. The list of exercises is shown in Table 2. Schematic representation of individual exercises is shown in Figure 2.

Table 2. The list and numbering of individual exercises performed by the subjects

\begin{tabular}{|c|c|c|}
\hline No. & Muscle & Type of exercise \\
\hline 1.1 & \multirow{7}{*}{ 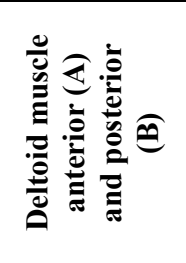 } & External rotation of arm \\
\hline 1.2 & & Internal rotation of arm \\
\hline 1.3 & & Barbell chin raise \\
\hline 1.4 & & Barbell raise in front of the head \\
\hline 1.5 & & Barbell raise behind the head \\
\hline 1.6 & & Barbell front raise \\
\hline 1.7 & & Lateral arm raise \\
\hline 2.1 & \multirow{4}{*}{ 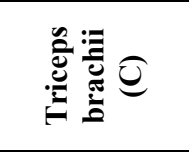 } & Winding under load \\
\hline 2.2 & & Wrist bend with barbell, overhand grip \\
\hline 2.3 & & Wrist bend with barbell, underhand grip \\
\hline 2.4 & & Wrist rotation \\
\hline 3.1 & \multirow{4}{*}{ 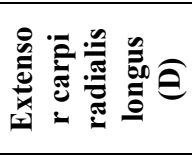 } & French press with both hands \\
\hline 3.2 & & Forearm extension \\
\hline 3.3 & & Barbell forearm extension, overhand grip \\
\hline 3.4 & & One arm French press \\
\hline
\end{tabular}

The obtained EMG traces were smoothed, and in the next step their RMS (Root Mean Square) values were determined. To normalize the results processed in this way it was necessary to determine a reference value. An arithmetic mean $(\bar{x})$ of the amplitude of the highest signal portion with $1000 \mathrm{~ms}$ fixed duration was defined as this value's estimator. From the values determined by this method the highest one was selected and it was subsequently used for the EMG signal normalization. The standard deviation of the arithmetic mean $\left(\sigma_{\bar{x}}\right)$ was adopted as the searched value error. For more detailed analysis of the recorded EMG traces, apart from the mean value, the median $(M e)$ was also determined as a parameter less sensitive to extreme values of the chosen portion of the amplitude. Normalization of the amplitude performed in the above-described manner does not change the nature of the EMG signal trace. It changes the $\mathrm{Y}$-axis scaling representing the signal as a percentage of the maximum measured value. The MVC test is one of the most commonly used methods of the EMG signal normalization [7-10]. It forms a basis for the comparison and evaluation of individual study results. In this manner a total of 198 mean amplitude values were determined (22 amplitude values from individual exercises for the analysed muscles for each of the 9 patients). 

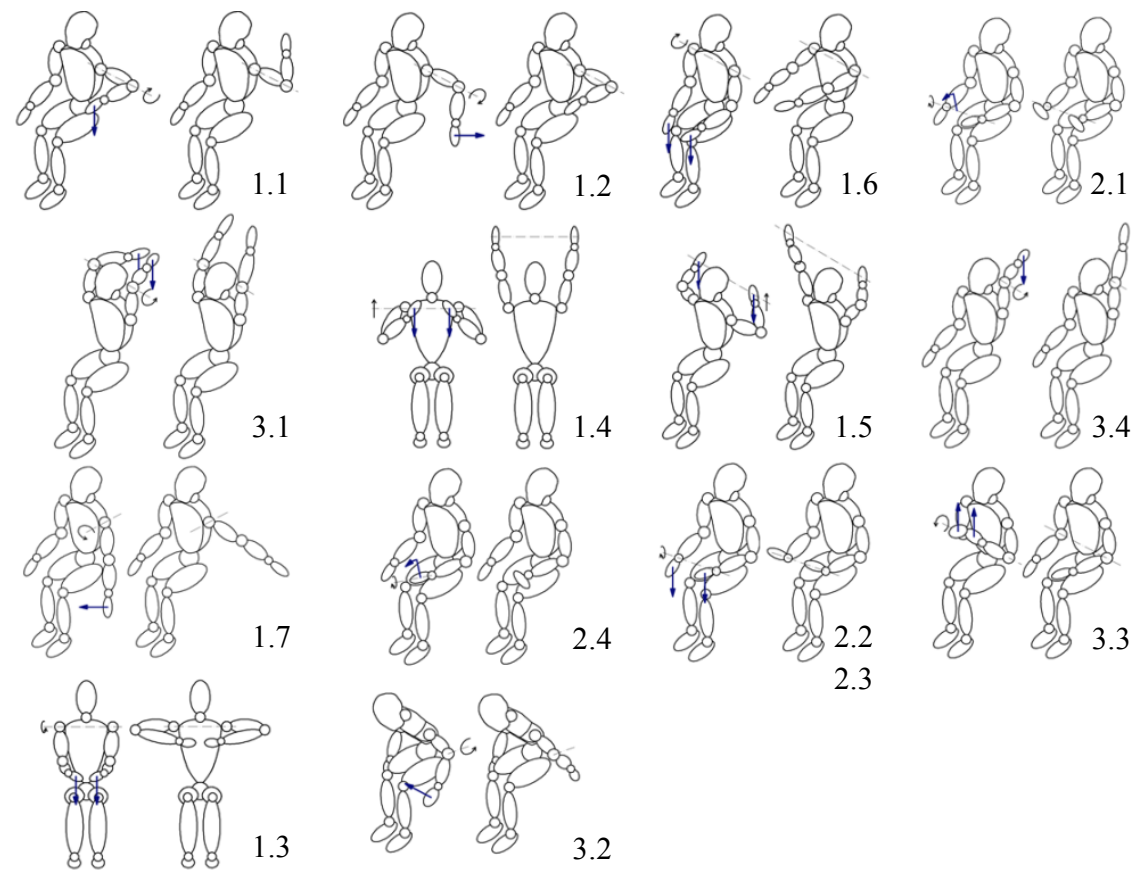

2.3

Fig. 2. Schematic representation of exercises performed by the subjects

\section{Results}

The research results were processed using the earlier-described methodology. The examples of the EMG traces measured and windows used are shown in Figure 3. Due to a large body of data collected only values for selected exercises and subjects are presented in a tabular form as Table 3. A graphic representation of the values determined is shown in Figure 4.

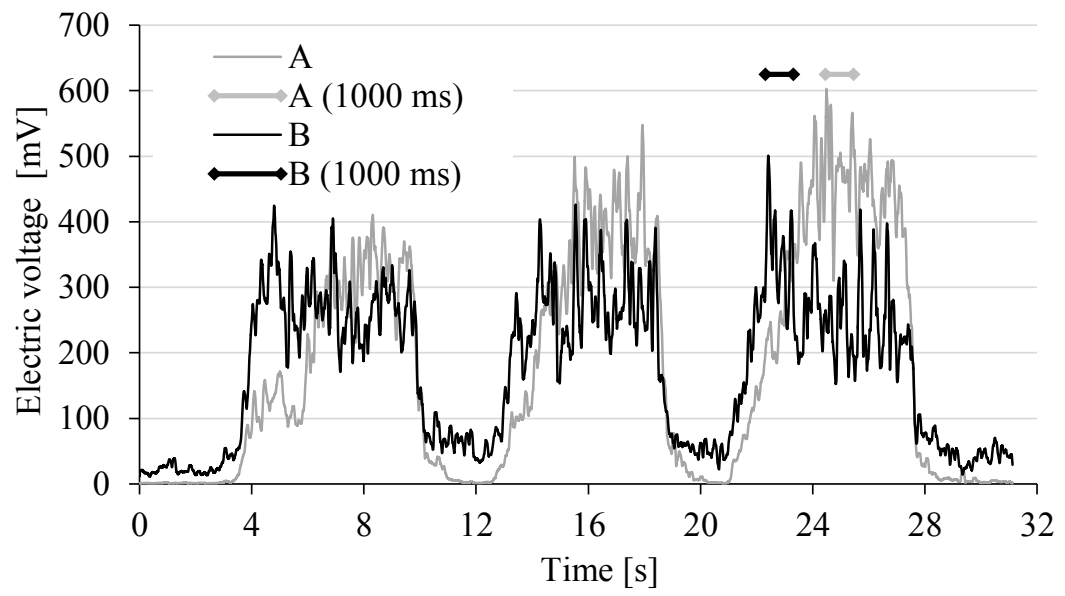

Fig. 3. Examples of measurement results for patient IV with selected $1000 \mathrm{~ms}$ signal windows marked, exercise: Lateral arm rise (1.7), deltoid muscle - anterior part (A) and posterior part (B) 
Table 3. Compilation of values determined for selected exercises and subjects

\begin{tabular}{|c|c|c|c|c|c|c|c|c|c|}
\hline \multicolumn{2}{|c|}{ Exercise } & \multicolumn{2}{|c|}{1.2} & \multicolumn{2}{|c|}{1.3} & \multicolumn{2}{|c|}{1.4} & \multicolumn{2}{|c|}{1.7} \\
\hline No. & Muscle & $\mathbf{A}$ & B & $\mathbf{A}$ & B & A & B & $\mathbf{A}$ & B \\
\hline \multirow{3}{*}{ I } & $\bar{x}$ & 23.6 & 293.0 & 16.2 & 219.7 & 49.5 & 142.1 & 60.7 & 145.0 \\
\hline & $\sigma_{\bar{x}}$ & 4.7 & 53.7 & 4.1 & 31.4 & 6.5 & 41.4 & 11.2 & 20.2 \\
\hline & Me & 24.4 & 292.9 & 15.9 & 216.6 & 48.7 & 126.9 & 59.1 & 144.1 \\
\hline \multirow{3}{*}{ III } & $\bar{x}$ & 141.1 & 473.8 & 265.4 & 290.5 & 273.5 & 38.2 & 365.6 & 270.4 \\
\hline & $\sigma_{\bar{x}}$ & 17.1 & 121.6 & 45.5 & 39.5 & 39.2 & 3.7 & 41.5 & 73.7 \\
\hline & Me & 143.5 & 459.8 & 265.6 & 285.5 & 272.0 & 38.1 & 365.5 & 283.5 \\
\hline \multirow{3}{*}{$\begin{array}{c}\text { VII } \\
\text { I }\end{array}$} & $\bar{x}$ & 206.5 & 94.7 & 94.1 & 61.8 & 183.1 & 34.6 & 428.5 & 30.7 \\
\hline & $\sigma_{\bar{x}}$ & 44.0 & 18.8 & 16.1 & 12.5 & 16.3 & 5.2 & 67.0 & 5.7 \\
\hline & Me & 197.3 & 97.9 & 96.9 & 60.7 & 184.1 & 34.6 & 435.1 & 30.9 \\
\hline \multirow{3}{*}{ IX } & $\bar{x}$ & 109.9 & 24.6 & 130.6 & 47.1 & 210.4 & 16.2 & 246.8 & 25.4 \\
\hline & $\sigma_{\bar{x}}$ & 15.8 & 4.9 & 16.0 & 6.8 & 20.7 & 2.2 & 39.3 & 4.7 \\
\hline & Me & 111.4 & 23.4 & 126.2 & 46.3 & 216.1 & 16.5 & 249.6 & 24.8 \\
\hline \multicolumn{2}{|c|}{ Exercise } & \multicolumn{2}{|l|}{2.1} & 2.2 & \multicolumn{2}{|c|}{2.4} & 3.1 & \multicolumn{2}{|r|}{3.2} \\
\hline No. & Muscle & \multicolumn{2}{|l|}{ C } & C & \multicolumn{2}{|c|}{$\mathrm{C}$} & D & \multicolumn{2}{|r|}{ D } \\
\hline \multirow{3}{*}{ I } & $\bar{x}$ & \multicolumn{2}{|c|}{272.7} & 270.4 & \multicolumn{2}{|c|}{99.5} & 25.1 & \multicolumn{2}{|r|}{34.8} \\
\hline & $\sigma_{\bar{x}}$ & \multicolumn{2}{|c|}{29.7} & 27.1 & \multicolumn{2}{|c|}{9.9} & 3.6 & \multicolumn{2}{|r|}{4.3} \\
\hline & Me & \multicolumn{2}{|c|}{273.6} & 267.9 & \multicolumn{2}{|c|}{99.5} & 24.3 & \multicolumn{2}{|r|}{34.1} \\
\hline \multirow{3}{*}{ III } & $\bar{x}$ & \multicolumn{2}{|c|}{307.0} & 333.0 & \multicolumn{2}{|c|}{96.1} & 236.9 & \multicolumn{2}{|c|}{260.5} \\
\hline & $\sigma_{\bar{x}}$ & 68.2 & & 39.9 & & & 46.9 & & 46.2 \\
\hline & Me & 293. & & 327.6 & & & 224.6 & & 245.5 \\
\hline & $\bar{x}$ & 171. & & 213.8 & & & 157.7 & & 274.0 \\
\hline VII & $\sigma_{\bar{x}}$ & 40.2 & & 20.3 & & & 23.8 & & 45.7 \\
\hline & Me & 164. & & 214.9 & & & 157.5 & & 270.7 \\
\hline & $\bar{x}$ & 66.5 & & 80.4 & & & 14.3 & & 20.0 \\
\hline IX & $\sigma_{\bar{x}}$ & 6.8 & & 42.4 & & & 2.1 & & 3.8 \\
\hline & Me & 65.3 & & 64.6 & & & 14.1 & & 19.7 \\
\hline
\end{tabular}

Analysis of the results obtained reveals big differences between mean amplitudes both for individual exercises and for individual study subjects. The standard deviation ranged from 10 to $30 \%$ of the mean value. These high values result both from the nature of the measurements and from the method used to determine the mean amplitude involving use of a time window of constant width of $1000 \mathrm{~ms}$. Additionally, a number of considerable deviations from a typical range of standard deviation values were observed. These included for instance: $\sigma_{\bar{x}}=49.8 \%$ (patient VI, exercise 1.2 , muscle A), $\sigma_{\bar{x}}=48.9 \%$ (patient V, exercise 1.4 , muscle B) and $\sigma_{\bar{x}}=65.5 \%$ (patient $\mathrm{V}$, exercise 2.3 , muscle C). The median and mean amplitude were similar for a great majority of the subjects. 


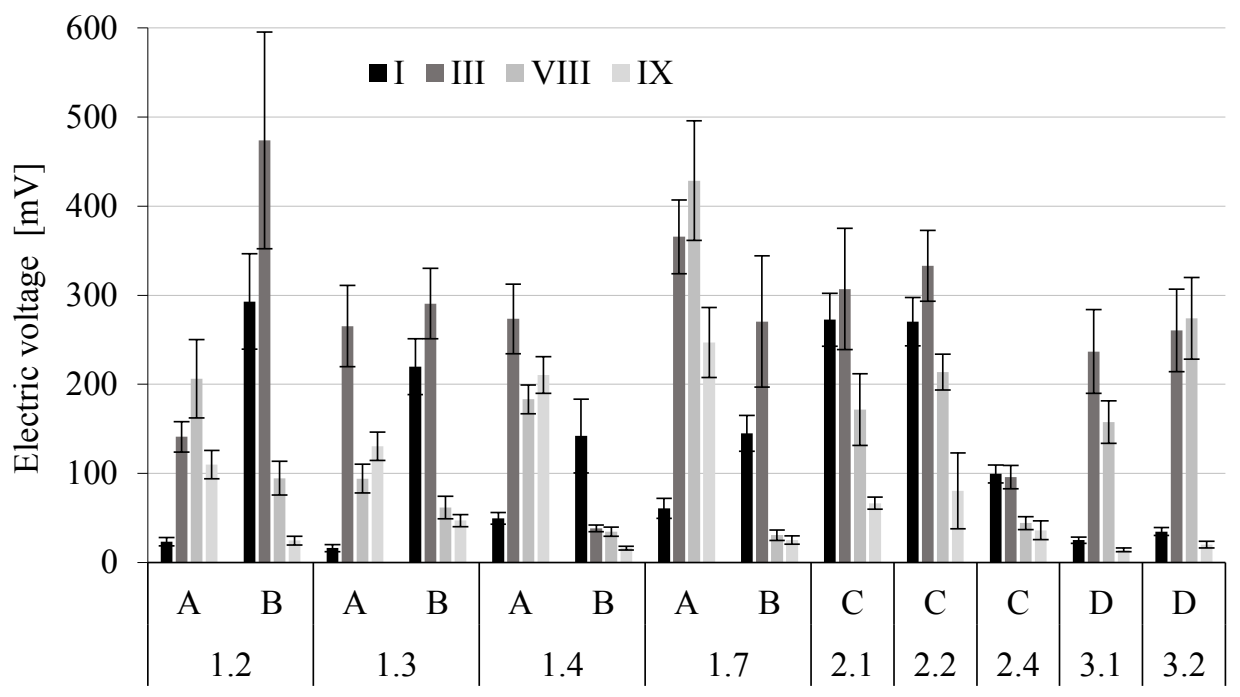

Fig. 4. Graphic representation of values obtained for selected exercises and subjects

Analysis of the data obtained revealed that big differences between these values occurred in cases of big measurement errors, that is, when considerable standard deviations were obtained. If the contamination of the EMG signal by potential artifacts was avoided or eliminated in a proper way, this can indicate non-static load application by the study subjects. Measurement results obtained in this manner cannot be used in further analyses. Thus, it is necessary to eliminate incorrectly determined measurement points or to repeat the test allowing to determine them again.

There are a number of limitations regarding the body mass index. This index cannot be used for people suffering from disease and children. Also attempts of classifications regarding sportsmen and women (for instance athletes and culturists) based on the BMI can lead to erroneous interpretations. No study subject can be classified as belonging to one of the abovementioned groups. Having this in mind, a relationship between the BMI value and the determined amplitudes can be observed. Usually, the greater the body mass index the lower the values of the calculated parameters. Human body is a relatively good conductor of electrical current. However, the conductance individual types of body tissues may be different and depends also on the thickness of a given layer. It is probably due to a greater thickness of the adipose tissue layer that the amplitudes obtained tend to be lower for persons with higher BMI values.

The analysis of all the results obtained revealed that exercises 2.1 and 2.2, chosen for the extensor carpi radialis longus, allow to obtain the most similar values of normalization amplitudes for all study subjects. Therefore, it can be stated that they are among the most effective resistance exercises necessary to conduct MVC tests under the given conditions of static contraction of muscles. In turn, the greatest spread of results was noted for exercise 2.3. From the technical point of view this exercise is performed correctly when the forearm is rotated in a way enabling grasping the intermediate element with underhand grip. In the process the belly of the investigated muscle moves under the electrodes. As a result small values, additionally characterized by a large spread, were obtained. Thus it can be concluded that this particular exercise is not useful for carrying out the normalization test. The greatest spread of the results for an individual muscle was observed during the tests regarding the triceps brachii. It was also observed that a large proportion of patients had problems with correct performance of exercises 3.1-3.4. It was concluded that the selected 
group of exercises for triceps brachii (D) should be significantly modified or new activities should be proposed.

\section{Conclusions}

Under this research project a number of MVC tests were performed for defined groups of muscles and exercises on a group of nine persons. For this purpose adequate procedures were developed, pertaining both to the preparation of patients for the tests and to the performance of the measurements. Suitable data processing methods as well as algorithms for determining the searched values were prepared. This formed a basis for an analysis of the parameters obtained, which allowed the authors to specify a group of the most effective resistance exercises required for the performance of the MVC tests under the set conditions of static muscle contraction. A group of exercises that should be rejected or modified was also indicated.

The study results and the experience gained from the investigation will allow the researchers in the future to increase the effectiveness of the works related to determining the activity of selected muscle groups during the performance of specific movements and exercises. This, in turn, will enable quality assessment of the biomechanics of propelling of manual wheelchairs of different types. The data obtained in the study form a basis for determining muscular activity occurring while propelling a wheelchair. The results will be compared and evaluated and will further serve as necessary input data for the design of innovative wheelchair drive systems to be used in vehicles intended for persons with physical disabilities.

The study was financed from the means of the National Centre for Research and Development under LIDER VII programme, research project no. LIDER/7/0025/L-7/15/NCBR/2016.

\section{References}

1. R. A. Cooper, D. P. VanSickle, R. N. Robertson, M. L. Boninger, G. J. Ensminger, A Method for Analyzing Center of Pressure During Manual Wheelchair Propulsion. IEEE Transactions on Rehabilitation Engineering 3 (3), 289-298 (1995)

2. M. L. Boninger, A. M. Koontz, S. A. Sisto, T. A. Dyson-Hudson, M. Chang, R. Price, R. A. Cooper, Pushrim biomechanics and injury prevention in spinal cord injury: Recommendations based on CULP-SCI investigations. Journal of Rehabilitation Research \& Development 42 (3), 9-20 (2005)

3. E. Świtoński, A. Głowacka, Określanie sit mięśniowych podczas chodu na podstawie sygnałów sEMG, Modelownie inżynierskie. t. 16 (47), 185-189 (2013)

4. D. Winter, H. Yack, EMG Profiles During Normal Human Walking: Stride-to-Stride and Inter-Subject Variability. Electroencephalography and Clinical Neurophysiology 67, 402-411 (1987)

5. Online at: http://www.seniam.org/

6. P. Konrad, „ABC EMG” Praktyczne wprowadzenie do elektromiografii kinezjologicznej. (Wyd. Technomex Sp. z o. o., ISBN 83-920818-1-1, Gliwice 2007)

7. B. Cestelein, B. Cagnie, T. Parlevliet, L. Danneels, A. Cools, Optimal Normalization Tests for Muscle Activation of the Levator Scapulae, Pectoralis Minor, and Rhomboid Major: An Electromyography Study Using Maximum Voluntary Isometric Contractions. Archives of Physical Medicine and Rehabilitation 96, (10), 1820-1827 (2015) 
8. H. Nieminen, E.-P. Taklal, E. Viikari-Juntura, Normalization of electromyogram in the neck-shoulder region. European Applied Journal of Physiology and Occupational Physiology 67 (3), 199-207 (1993)

9. R. A. Ekstrom, G. L. Soderberg, R. A. Donatelli, Normalization procedures using maximum voluntary isometric contractions for the serratus anterior and trapezius muscles during surface EMG analysis. Journal of Electromyography and Kinesiology 15 (4), 418-428 (2004)

10. M. Halaki, K. Ginn, Normalization of EMG Signals: To Normalize or Not to Normalize and What to Normalize to?, In: Computational Intelligence in Electromyography Analysis - A Perspective on Current Applications and Future Challenges, Chapter 7. (Publisher: Rijeka, InTech, ISBN 978-953-51-0805-4, 2012) 